\title{
Política sanitaria autonómica y Cohesión territorial
}

\section{$M^{a}$ Jesús Montero Cuadrado}

Arbor CLXXX, 710 (Febrero 2005), 375-382 pp.

Hace ya dos años que fue aprobada la Ley de Cohesión y Calidad del SNS, que, en teoría, debía ir dirigida a garantizar la coordinación en un sistema sanitario completamente descentralizado. Sin embargo la realidad es obstinada, y los acontecimientos más recientes nos demuestran que aún queda mucho camino por recorrer para garantizar que disponemos de un Sistema Nacional de Salud coordinado y, sobre todo, que siguen vigentes los principios básicos establecidos en la ley de Sanidad y la citada ley de Cohesión, es decir, que todos seguimos apostando por un modelo de sistema nacional de salud que garantice la cohesión territorial.

Entendiendo éste, el SNS, como uno de los elementos básicos de la Sociedad del Bienestar, debemos seguir trabajando para que sea un Sistema equitativo, coordinado, y, sobre todo, que no rompa la cohesión y el equilibrio territorial entre todos los ciudadanos de este país. Para ello es imprescindible seguir manteniendo muy claras las reglas del juego, y compatibilizar la necesaria descentralización con la coherencia de un Sistema que, además de otras muchas funciones, debe responder también a la de redistribuir la riqueza en una sociedad en la que, todavía, las diferencias interterritoriales siguen siendo de una gran magnitud.

\section{El papel del estado}

Las comunidades autónomas tenemos que seguir desempeñando el papel que nos corresponde, pero el Estado tiene también que jugar un rol muy importante en este sistema descentralizado, pues debe fijar el mar- 
co regulador, garantizar la cobertura universal y el aseguramiento único y público, acreditando que funcionen los mecanismos de solidaridad. Creemos que estos elementos son tan importantes, que exigen un Ministerio de Sanidad fuerte, con atribuciones suficientes para ello. Sin embargo, cuando se promulgó la Ley de Cohesión y Calidad se perdió la oportunidad de dotar al Estado de los mecanismos adecuados para garantizar la equidad del sistema.

Además de este papel, el Estado tiene también otras muchas responsabilidades; pero me gustaría insistir en que una de las más determinantes es asegurar mecanismos de financiación que estén sustentados en la corrección de desigualdades.

Todo ello debe ejercerse desde una actitud consecuente con la realidad de un Estado plural, yo me atrevería a llamarlo cuasi-federal, ejerciendo su propio papel y favoreciendo el correcto desarrollo de las competencias de las comunidades, sin interferencia y sin dejación de responsabilidades. En este marco, la coordinación territorial de las políticas de salud se convierte en uno de los retos más difíciles que tenemos por delante. Desde Andalucía creemos que es posible compatibilizar el proceso de descentralización con la profundización en la igualdad y solidaridad entre los territorios. Para ello, la coordinación debe ser entendida como un mecanismo multilateral, no unidireccional (del centro a la periferia); debe ser una tarea de todos los responsables del gobierno sanitario, porque todos somos Estado, y debe estar plenamente integrada en el papel del Consejo Interterritorial.

Respecto a este último, creo que resulta imprescindible reforzar su carácter ejecutivo, modificando su capacidad de adoptar decisiones. Habrá que determinar en qué ámbitos deben y pueden tomarse por consenso y en cuáles a través de un sistema de voto ponderado. $\mathrm{Y}$, por supuesto, es necesario crear una disciplina común de seguimiento de los acuerdos adoptados.

Tenemos algunos problemas básicos de coordinación en los que, sin duda, el papel del Consejo Interterritorial, así como de otras instancias en casos determinados, debería ser clave: las políticas de profesionales, la política farmacéutica y la financiación del sistema.

\section{Políticas coordinadas}

Respecto a la primera, las políticas de profesionales, es indudable que cada comunidad debe marcar el modelo de crecimiento y desarrollo profesional que le parezca más adecuado a sus necesidades, siempre en 
el marco de las leyes del Estatuto y de Ordenación de las Profesiones Sanitarias. Pero también es evidente que no podemos mantener, porque a ninguna comunidad nos interesa, la espiral de crecimiento, para muchos insostenible, a la que nos estamos viendo sometidos. Y el Consejo debería debatir, y pronunciarse, sobre algunos asuntos clave: ¿están mal retribuidos los profesionales sanitarios? ¿cómo debe ser la carrera profesional para garantizar la movilidad territorial? ¿podemos buscar otros modelos retributivos que incentiven mejor al profesional? ¿tendríamos que establecer otro tipo de relación contractual?.

Otro elemento básico de coordinación es el que se refiere a las políticas de farmacia. Es necesario, imprescindible, reconducir el crecimiento del gasto farmacéutico a niveles compatibles con su sostenibilidad. Habida cuenta de que la falta de una selección racional de medicamentos y de un modelo de financiación y precios coherente con su utilidad, es uno de los motivos que los expertos identifican como causa de las elevadas tasas de crecimiento del gasto farmacéutico público, abogamos por la necesidad de participar de forma activa en las decisiones que en éste sentido deben todavía adoptarse.

Por ello nos parece que debe ser el Consejo Interterritorial el que proponga la normativa necesaria para la regulación de los nuevos criterios de selección, calificación y precios máximos de los medicamentos que vayan a ser financiados por el Sistema Nacional de Salud. Y, en el mismo sentido, las normas reguladoras de los nuevos criterios de fijación de precios industriales (ligados a utilidad terapéutica y ventas) y retribución del canal farmacéutico.

De igual modo coincidimos en que los criterios que deben regir la selección de los medicamentos deben ser los de utilidad terapéutica demostrada y comparada, así como su necesidad real en el SNS. De esta manera, será clave el papel del Consejo Interterritorial, garantizando que se pongan a disposición de la financiación pública sólo aquellos medicamentos necesarios y al precio más ajustado posible.

Si no se produce esta adaptación normativa y reguladora, creemos que el Estado debe asumir una parte de la financiación del gasto farmacéutico, al no permitir que las comunidades tengamos capacidad real en la decisión de qué medicamentos se pueden financiar a través de las prestaciones públicas del SNS. De igual modo, he apuntado en alguna ocasión la posibilidad de que la seguridad social se haga cargo de la factura farmacéutica de los pensionistas, al ser una prestación más de entre las que oferta para sus 'mutualistas'.

Junto con estas medidas, sería necesaria también una nueva política en $\mathrm{I}+\mathrm{D}+\mathrm{i}$ que comprometa seriamente a la Industria y que proteja sólo la 
verdadera innovación terapéutica, así como favorecer los mecanismos para que las comunidades tengamos herramientas efectivas de control y uso racional.

En Andalucía, por ejemplo, decidimos en su momento realizar la prescripción farmacéutica por principio activo (y hoy superamos de esta forma el $60 \%$ de la prescripción); nuestro objetivo va a más, y es llegar incluso al equivalente terapéutico; para ello estamos dedicando, desde hace muchos años, un volumen importante de nuestro tiempo y nuestro esfuerzo a diseñar políticas de uso racional del medicamento, y a trabajar con los médicos en la implantación de estas medidas.

Me refiero a continuación a otro tema clave de coordinación, no sólo en nuestra agenda sino en la de los presidentes autonómicos y la de toda la sociedad: la financiación de la sanidad.

\section{Financiación sanitaria}

Creemos que el actual modelo de financiación de las comunidades es un modelo válido, aunque susceptible de posibles ajustes en lo que afecta a la financiación de la sanidad. El primero de ellos sería determinar la cuantía adecuada para que la financiación sanitaria de las comunidades sea suficiente. Partimos de una situación de insuficiencia estructural del modelo, carencia que debería compartir el Estado una vez acordado cómo calcular su cuantía para las distintas comunidades autónomas. Porque lo que no es equitativo es hacer 'tabla rasa'. Ello perjudica a las comunidades más eficientes, que, en un claro ejercicio de responsabilidad, hemos conseguido no desviarnos de los mínimos marcados.

Aunque el SNS es un sistema eficiente, existen todavía márgenes que permiten racionalizar el gasto sin disminuir la eficacia. Estos márgenes son además muy variables de unas comunidades a otras. En un sistema público como el nuestro nunca debemos obviar el principio ético de la eficiencia: obtener la mayor rentabilidad social sobre los recursos que la sociedad deposita en nuestras manos.

Otro aspecto clave del modelo de financiación son las variables y ponderaciones que se utilicen para calcular las necesidades de financiación de cada comunidad. El modelo actual distribuye los recursos de acuerdo al tamaño de la población. Y considero que es un criterio equitativo. Pero las correcciones para ajustar dicha base y paliar desigualdades pueden hacer perder equidad si no están suficientemente ponderadas. Por ello proponemos que se tengan en cuenta otros criterios sanitarios y socioeconómicos además de los actuales. 
Es necesario también abordar el origen de los recursos destinados a financiar la sanidad. En Andalucía no somos partidarios de las fórmulas de participación directa de los ciudadanos por el acceso o uso de los servicios sanitarios que hoy están reconocidos en nuestras carteras de servicios, y que forman parte de los elementos básicos de la atención sanitaria. Con estas medidas se podría penalizar de forma injusta a pacientes crónicos y ancianos; es más, no existe evidencia de que estos mecanismos de participación directa del usuario supongan un elemento de disuasión real al uso abusivo de la sanidad. Por otra parte, los previsibles costes de gestión de esas medidas minimizarían el supuesto volumen de recursos generado.

Otra posibilidad que quizás sí cabría en este debate sería utilizar alguna de éstas fórmulas de participación de cara a la puesta en marcha de medidas que vayan más allá de la atención sanitaria básica. Medidas que supongan un plus adicional en la calidad de las prestaciones o que éstas sean claramente diferenciadas de la atención sanitaria (sería el caso, por ejemplo, de determinadas prestaciones sociosanitarias).

Las cesiones de impuestos, subidas impositivas o creación de más tasas e impuestos, son cuestiones a tratar con mucha precaución, siempre desde la óptica de la equidad del sistema, la vertebración y la cohesión social, que, en cualquier caso, siempre deberían quedar garantizadas. No podemos olvidar que alguno de estos aspectos pudieran tener una clara repercusión negativa en las comunidades menos ricas.

Teniendo en cuenta que lo que sí es indudable es la necesidad de un incremento de la financiación general del sistema, que permita la potenciación de elementos de cohesión y redistribución de recursos, consideramos de vital importancia la potenciación del Fondo de Cohesión Sanitaria o el establecimiento de algún tipo de fondo destinado a corregir desigualdades en salud.

El debate, pues, está servido. Presenta múltiples componentes y variantes para la discusión, y, sin duda, es clave para garantizar la cohesión y sostenibilidad del sistema nacional de salud tal como fue concebido hace ya 20 años. Y nos encontramos cada vez con nuevos elementos de discusión y preguntas clave. ¿El Estado tiene que dotarse de los mecanismos adecuados para controlar la actuación eficiente de las comunidades a través de los recursos que éstas perciben?, ¿cómo se articulan estos mecanismos de control? y, sobre todo, ¿qué papel debe jugar el Estado, una vez que estos mecanismos pudieran poner de manifiesto el comportamiento 'poco eficiente' de determinadas comunidades?

Un último comentario sobre la financiación, en aspectos directamente relacionados con los principios éticos. Las comunidades tenemos capa- 
cidad real de autogobierno, y, por supuesto, de ampliar, mejorar o añadir aquellas prestaciones que consideremos necesarias. Son los propios parlamentos regionales los que, en su ejercicio soberano, pueden decidir la asignación de sus partidas presupuestarias.

En esta línea argumental, creo que las comunidades debemos asumir que la financiación de todo lo que no esté pactado dentro de los mínimos de la cartera de servicios común para el Estado debe ser responsabilidad propia. Nunca puede entrar a formar parte del cálculo de la insuficiencia y la necesidad global de financiación.

Pero, en el mismo sentido, debe ser totalmente asumida la política de corresponsabilidad financiera del Estado con las comunidades autónomas. No se pueden imponer nuevas prestaciones o adoptar regulaciones, desde el nivel central, que comporten incremento de gastos, si no se adapta y aporta la financiación adecuada para ello. Y llámense prestaciones o llámese también nuevos reconocimientos profesionales, nuevas titulaciones o, con un ejemplo muy reciente, nuevas especialidades sanitarias.

\section{La política sanitaria andaluza}

Desde la comunidad andaluza creemos que la financiación sanitaria es un tema de crucial importancia para garantizar la sostenibilidad de nuestro sistema sanitario público. Pero también sabemos que no es el único, y que, entre todos, debemos contribuir a su legitimidad y sostenibilidad poniendo en marcha todos los instrumentos posibles y necesarios, instrumentos que forman parte ineludible de nuestra agenda política. Ya en la Ley 2/98 de Salud de Andalucía planteamos la necesidad de orientar nuestras políticas hacia el ciudadano, y en este sentido configuramos una nueva arquitectura de derechos, de forma que nuestro sistema pasó de ser un sistema de mera prestación de servicios a un sistema garante de derechos ciudadanos.

Esto ha significado, en estos años, ir más allá de la universalización y gratuidad, y plantear estos nuevos derechos ciudadanos, reconociéndolos normativamente. Así, junto a la libre elección de médico y hospital, hemos desarrollado el derecho a una segunda opinión médica, el derecho a otorgar la voluntad vital anticipada, el derecho a las garantías de tiempos máximos de espera quirúrgica así como de un tiempo máximo de espera diagnóstica, en consultas externas y procesos asistenciales prioritarios. En esta legislatura estamos ampliando esta arquitectura de derechos, siempre con el objetivo de que el ciudadano considere el sistema sanitario público como un bien irrenunciable. 
Pero además trabajamos intensamente en aras de la eficiencia del sistema, pues como comenté más arriba, no podemos solicitar financiación adicional si no ponemos en marcha toda una batería de medidas propias que nos ayuden a gestionar el sistema público cada vez mejor. Son medidas dirigidas a mejorar la eficiencia en la gestión de los recursos, de las personas, de la producción asistencial y de la propia organización.

Entre las primeras podemos señalar elementos como la Central Logística de Compras, que ordena y racionaliza todas las compras de la organización vía Web, o los sistemas de contabilidad analítica o de información de recursos humanos; ambos posibilitan la medición de costes por servicios y procesos, y permiten conocer dónde y en qué se gasta, y hacer limitativo el presupuesto de cada centro.

Otros instrumentos que también se traducen en una indudable eficiencia en la gestión de los recursos, aunque no han sido concebidos para este fin, son la Historia de Salud Electrónica y la Receta Electrónica. $\mathrm{Al}$ ser una historia digital única por cada paciente, accesible desde cualquier punto del sistema, evita traslado de papel, repetición de pruebas (y consumos) e, indudablemente, mejora la calidad de la atención.

Desde el punto de vista de la gestión de las personas, los modelos de gestión por competencias y desarrollo profesional, recientemente puestos en marcha, así como las entrevistas de desarrollo profesional y la determinación de los complementos de rendimiento, permiten orientar las actuaciones de los profesionales a objetivos de eficiencia, no sólo económica sino, en primer lugar, eficiencia social con mejores resultados en salud.

De igual modo, y para finalizar, todas las medidas dirigidas a conseguir una nueva forma de organización, más horizontal, que trabaje orientada a resultados en salud y garantizando la continuidad asistencial, se traducen también en mejora de la eficiencia. Me refiero aquí a nuevas formas organizativas como las Unidades de Gestión Clínica o la Gestión por Procesos, así como a la potenciación de otras medidas con un impacto indudable en la mejora de la calidad asistencial pero también en la eficiencia del sistema y la satisfacción del ciudadano. Es el caso de las consultas de acto único y los centros de alta resolución, la Telemedicina, o las formas alternativas a la hospitalización tradicional, como la cirugía mayor ambulatoria o los hospitales de día.

En definitiva, desde hace más de 20 años, mantenemos una agenda y pensamiento político concretos, que creemos que nos ha permitido ir caminando en la dirección adecuada. En el momento actual, y con la puesta en marcha del II Plan de Calidad, nos planteamos la estrategia política de innovación, modernización y transformación del sis tema. Y no sólo para mejorar su calidad, sino también para responder 


\section{$M^{a}$ Jesús Montero Cuadrado}

382

al crecimiento de las demandas y expectativas de los ciudadanos. Sólo así seguiremos construyendo un sistema sanitario público sostenible y legítimo. 